\title{
Development and verification of a risk prediction model for bronchopulmonary dysplasia in very low birth weight infants
}

\author{
Huiwen Cai ${ }^{1}$, Ling Jiang ${ }^{2}$, Yongshu Liu ${ }^{1}$, Ting Shen ${ }^{1}$, Zuming Yang ${ }^{1}$, Sannan Wang ${ }^{1}$, Yuelan Ma ${ }^{1}$ \\ ${ }^{1}$ Division of Neonatology, Suzhou Municipal Hospital, The Affiliated Suzhou Hospital of Nanjing Medical University, Suzhou, China; ${ }^{2}$ Nursing \\ Department, Suzhou Municipal Hospital, The Affiliated Suzhou Hospital of Nanjing Medical University, Suzhou, China \\ Contributions: (I) Conception and design: Y Ma; (II) Administrative support: H Cai, L Jiang; (III) Provision of study materials or patients: Y Liu, T \\ Shen; (IV) Collection and assembly of data: Z Yang; (V) Data analysis and interpretation: S Wang; (VI) Manuscript writing: All authors; (VII) Final \\ approval of manuscript: All authors. \\ Correspondence to: Yuelan Ma. Division of Neonatology, Suzhou Municipal Hospital, The Affiliated Suzhou Hospital of Nanjing Medical University, \\ Suzhou 215000, China. Email: mayuelan2021@163.com.
}

Background: To analyze the risk factors of bronchopulmonary dysplasia (BPD) of very low birth weight infants (VLBWIs), and to develop and verify a risk prediction model of BPD.

Methods: The data of 611 VLBWIs from the neonatal intensive care unit (NICU) of a tertiary grade A hospital in Suzhou from January 2017 to September 2019 were collected. The data was randomly divided into the modeling set (451 cases) and the validation set (160 cases). Binary logistic regression was used to analyze the data, and the model was examined by a receiver operating characteristic (ROC) curve. The grouped data was used to verify the sensitivity and specificity of the model.

Results: The study found that neonatal asphyxia, the positive rate of sputum culture, neonatal sepsis, neonatal respiratory distress syndrome (NRDS), blood transfusions ( $\geq 3$ ), patent ductus arteriosus (PDA), the time of invasive mechanical ventilation, the duration of oxygen therapy, and the time of parenteral nutrition were the independent risk factors of BPD, while $1 \mathrm{~min}$ Apgar score was a protective factor. The model formula was $Z=$ neonatal asphyxia * $1.229+$ the positive rate of sputum culture * $1.265+$ neonatal sepsis * $1.677+$ NRDS * $1.848+$ blood transfusions $(\geq 3) * 1.455+$ PDA * $1.835-1$ min Apgar score * $0.25+$ the time of invasive mechanical ventilation * $0.123+$ the duration of oxygen therapy * $0.09+$ the time of parenteral nutrition * $0.057-8.077$. The area under the ROC curve of this model was 0.965 (95\% CI: 0.946-0.983), with a sensitivity of $93.7 \%$ and a specificity of $91.3 \%$. Verification of this prediction model showed a sensitivity of $92.9 \%$ and a specificity of $76 \%$, demonstrating that the effects of this model were satisfactory.

Conclusions: The risk prediction model had a good predictive effect for the risk of BPD in VLBWIs, and can provide a reference for preventive treatment and nursing intervention.

Keywords: Very low birth weight infant (VLBWI); bronchopulmonary dysplasia (BPD); risk prediction model

Submitted Aug 13, 2021. Accepted for publication Oct 19, 2021.

doi: $10.21037 / \mathrm{tp}-21-445$

View this article at: https://dx.doi.org/10.21037/tp-21-445

\section{Introduction}

Bronchopulmonary dysplasia (BPD) refers to the need for oxygen or respiratory support at least 28 days after birth (1). BPD is a common complication of premature infants, especially very low birth weight infants (VLBWIs) or extremely low birth weight infants (ELBWIs). The preterm delivery of VLBWIs could be induced by chorioamnionitis or sepsis. Further evidence confirms sepsis could induce BPD in VLBWIs. Chorioamnionitis is not the etiology of BPD (2). Early systemic inflammation has been proved to induce BPD in ELBWIs. Serum eotaxin level is a good marker in predicting BPD associated death at birth (3). BPD can easily cause extremely high morbidity and mortality of related diseases, and seriously threatens the 
lives and the later quality of life of children (4). ValenzuelaStutman et al. reports $15.7 \%$ of the VLBWIs and $37.3 \%$ of the VLBWIs were dead. At birth, body weight and gestation age are the main influence factors of BPD related death, while the length of ventilation and oxygen treatment are the main influence factors of BPD related death (5). With the development of perinatal medicine and the improvements in neonatal intensive care technology, the survival rate of VLBWIs/ELBWIs is gradually increasing. However, the incidence of BPD is constantly increasing and the degree of BPD is becoming more and more serious (6-8). Children with BPD generally have a poor prognosis and are prone to various sequelae, such as recurrent respiratory tract infection, reactive airway disease, growth disorders, and developmental delay, among others, and no specific treatment has been found in the clinical scope $(9,10)$. Therefore, it is necessary to actively explore tools for predicting the occurrence of BPD in VLBWIs, so as to help medical personnel assess and implement early treatment and nursing measures to reduce the adverse effects of BPD. As BPD could be induced by multi-factors, evidences have reported some related risk factors of $\mathrm{BPD}$, like intrauterine growth retardation, ventilation length, hyperoxia treatment, infection, genetic susceptibility, chorioamnionitis and preeclampsia $(11,12)$. Besides these factors, exosomal $\mathrm{miR}-876-3 \mathrm{p}$ is been reported to predict and protect BPD in premature infants (13). Compared with VLBWIs without BPD, the level of circulating endostatin is higher in VLBWIs with BPD, which indicating the correlation between endostatin and BPD (14). Foreign risk prediction models are not applicable to Chinese children (4), and there is no domestic prediction model for the occurrence of BPD in VLBWIs. Therefore, the purpose of this study is to build a risk prediction model for BPD occurrence in VLBWIs, and to provide a basis for predicting and managing the risk of BPD occurrence in VLBWIs. We present the following article in accordance with the STARD reporting checklist (available at https://dx.doi.org/10.21037/tp-21-445).

\section{Methods}

\section{Subjects}

The present study was a single-center retrospective study. Using the convenience sampling method, VLBWIs with complete case data from the neonatal intensive care unit (NICU) of a tertiary grade A hospital in Suzhou from January 2017 to September 2019 were included.
The inclusion criteria were as follows: (I) body weight $\leq 1,500 \mathrm{~g}$ and gestational age $<32$ weeks; (II) preterm infants with survival time $\geq 28$ days. The exclusion criteria were as follows: (I) severe congenital malformations such as chromosomal abnormalities, congenital pulmonary disease, congenital heart disease (except patent ductus arteriosus (PDA) and atrial septal defect), and central nervous system malformations; (II) those who were critically ill and died after their families gave up treatment automatically; (III) incomplete clinical data and lost to follow-up. In this study, the logistic events per variable (EPV) method was used to calculate the sample size of the modeling data (15). When the Wald method is used, the EPV should be at least $>5$ to ensure the stability of the results. In this study, risk factors were screened by univariate analysis and multivariate analysis, and 10 covariables were included in the final model. The incidence of BPD was $14 \%(7 / 50)$ in 50 samples of our hospital. The EPV was 5 . The required sample size of the modeling data was $(10 \times 5) \div 14 \%=357$ cases. Finally, 611 infants were included in this study and were randomly divided into the modeling group (451 cases) and the validation group (160 cases) according to 7:3 systematic sampling. The modeling group was used for the establishment of BPD risk prediction models, and the validation group was used for the external validation of models. All procedures performed in this study involving human participants were in accordance with the Declaration of Helsinki (as revised in 2013). The study was approved by ethics committee of Suzhou Municipal Hospital (No. K-2020-053-K01) and informed consent was taken from the patients' parents or legal guardians.

\section{Research method}

\section{Research tools}

The researchers used literature research, research group discussions, expert group meetings, and other methods to compile questionnaires on the factors related to the occurrence of BPD in VLBWIs. These mainly included: (I) general clinical data of the infants: gender, birth gestational age, birth weight, delivery mode, multiple birth, small for gestational age (SGA), in vitro fertilization, intrauterine distress, Apgar score, hospitalization days; (II) maternal general condition: maternal age, history of premature rupture of membranes, gestational hypertension, gestational diabetes, placental abnormalities (placenta previa and placental abruption), etc.; (III) treatment during hospitalization: invasive mechanical ventilation time, continuous positive airway pressure (CPAP) time, whether 
a pulmonary surfactant (PS) was used, whether caffeine/ aminophylline was used, number of blood transfusions, total days using oxygen, duration of parenteral nutrition, duration of enteral nutrition, whether sputum culture was positive, etc. (IV) main diseases and complications after birth: neonatal respiratory distress syndrome (NRDS), neonatal asphyxia, clinical septicemia, neonatal hyperbilirubinemia, PDA, necrotizing enterocolitis (NEC), retinopathy of prematurity (ROP), intraventricular hemorrhage (IVH), anemia in premature infants, neonatal hypoglycemia, etc.

\section{Data collection method}

The required data were obtained by retrospectively investigating the medical records from the hospital's electronic medical record system, nursing document system, and medical record room. The data was double checked and input using Excel software. Before the formal investigation, the research group conducted professional training for the investigators, and carried out the investigation after passing the assessment. After the survey was completed, special personnel kept the data and checked $5-10 \%$ of the patients' data to ensure its accuracy.

\section{Statistical analysis}

The measurement data conforming to the normal distribution were expressed as mean \pm standard deviation $\left(\bar{x}_{ \pm s}\right)$. The independent-samples $t$-test was used for intergroup comparisons. Measurement data with a non-normal distribution were described by median and quartile, and the Wilcoxon signed rank test was used to compare 2 independent samples. Count data were expressed as frequency and percentage, and the $\chi^{2}$ test or Fisher's exact probability test was used for comparisons between groups. The predictive model was constructed using binary logistic regression and forward selection (likelihood ratio), the inclusion and exclusion criteria were 0.05 and 0.10 , respectively, and the Hosmer-Lemeshow (H-L) test was used to verify the fitting degree of the model. The larger the $\mathrm{P}$ value, the better the fitting degree. The prediction ability of the model was evaluated by the area under the receiver operating characteristic (ROC) curve, and $\mathrm{P}<0.05$ was considered statistically significant.

\section{Results}

\section{General information of the patients}

A total of 611 cases were included, including 315 males
(51.6\%) and 296 females (48.4\%). The mean gestational age was $29.92 \pm 2.14$ weeks, the mean birth weight was $1,210.11 \pm 194.26 \mathrm{~g}$, the mean hospital stay was $39.07 \pm 20.07$ days, and the 1-minute Apgar score was $7.78 \pm 2.19$. There were 473 single births (77.4\%), 138 multiple births (22.6\%), and 70 in vitro fertilization cases (11.5\%).

\section{Results of the univariate analysis of general data between the 2 groups of infants in the modeling group}

A total of 451 cases in the modeling group were divided into the BPD group and the non-BPD group. Among them, 63 cases were in the BPD group, and the incidence of BPD was $14.9 \%$. There were statistically significant differences in 24 variables such as sex, gestational age at birth, birth weight, and Apgar score $(\mathrm{P}<0.05)$ (Table 1).

\section{The establishment of the risk prediction model}

Variables with statistically significant differences in the univariate analysis were taken as independent variables, and the dependent variable of the occurrence of BDP was analyzed by binary logistic regression. The variable assignment is shown in Table 2. The results of this study showed that neonatal asphyxia, positive sputum culture, clinical septicemia, NRDS, blood transfusion $\geq 3$ times, PDA, invasive mechanical ventilation time, oxygen time, and parenteral nutrition time were independent risk factors, and 1-minute Apgar score was a protective factor, as shown in Table 3. Model formula: $Z=$ neonatal asphyxia * $1.229+$ positive sputum culture * $1.265+$ clinical septicemia ${ }^{*} 1.677$ + NRDS * $1.848+$ number of blood transfusions $(\geq 3) * 1.455$ + PDA*1.835 - 1 min Apgar score * 0.25+ time of invasive mechanical ventilation * $0.123+$ time of oxygen application * $0.09+$ parenteral nutrition * 0.057 to 8.077 .

\section{Test of fitting degree and analysis of the prediction effect of the risk prediction model}

The H-L test was performed to verify the fitting effect of the prediction model, and the results showed that the model could fit the actual occurrence of BPD in VLBWIs $(\mathrm{P}=0.983)$. The cut-off value calculated by the prediction model formula was used as the test variable, and the occurrence of BPD was used as the state variable to draw the ROC curve. The results in Figure 1 show that the area under the ROC curve was 0.965 (95\% CI: $0.946-0.983, \mathrm{P}<0.001$ ), the sensitivity was $93.7 \%$, and the specificity was $91.3 \%$, indicating that the predictive 
Table 1 Univariate analysis of risk factors for BPD in VLBWIs

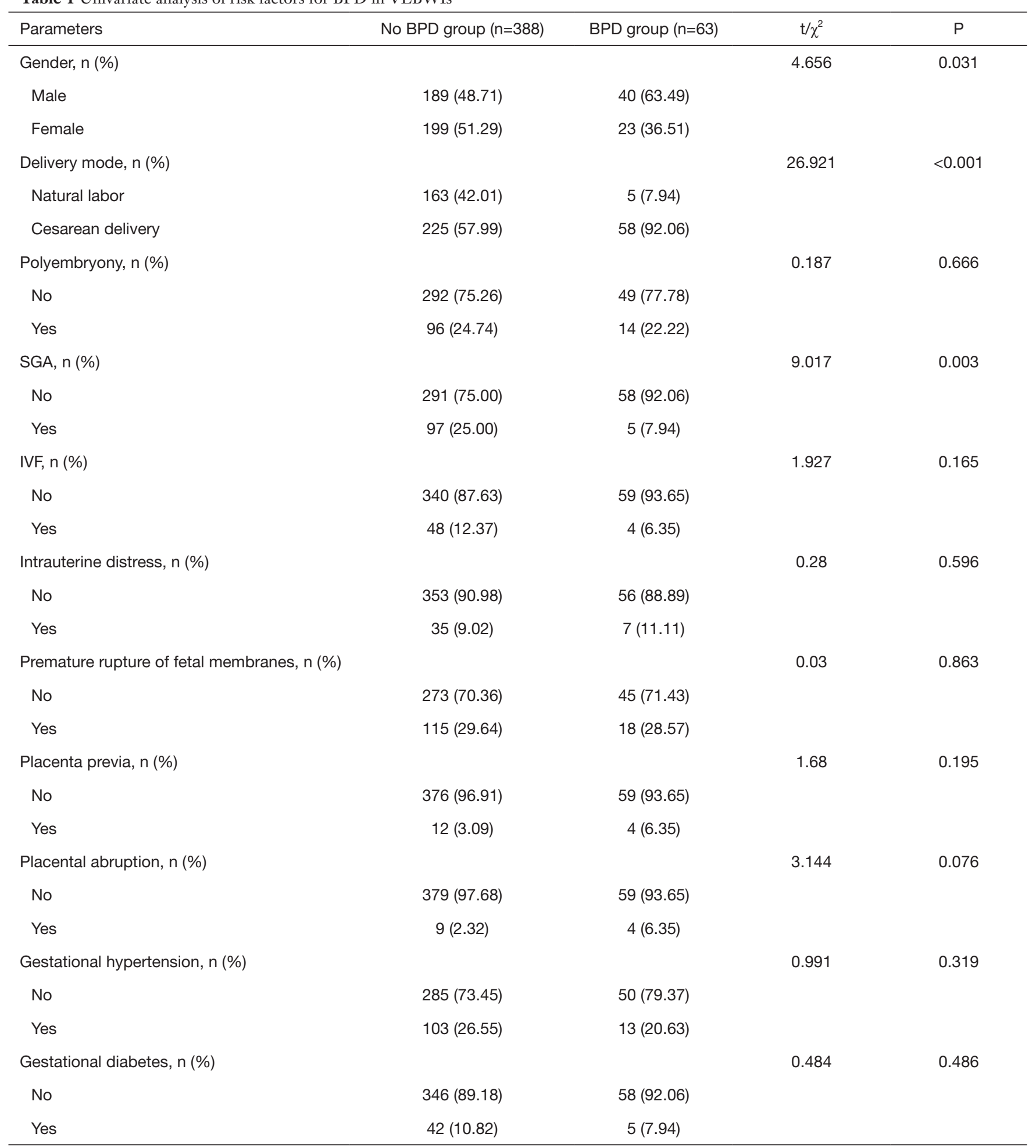

Table 1 (continued) 
Table 1 (continued)

\begin{tabular}{|c|c|c|c|c|}
\hline Parameters & No BPD group $(\mathrm{n}=388)$ & BPD group $(n=63)$ & $t / \chi^{2}$ & $\mathrm{P}$ \\
\hline No & $142(36.60)$ & $29(46.03)$ & & \\
\hline Yes & $246(63.40)$ & $34(53.97)$ & & \\
\hline PS use, n (\%) & & & 33.03 & $<0.001$ \\
\hline Yes & $157(40.46)$ & $50(79.37)$ & & \\
\hline Caffeine/aminophylline, $\mathrm{n}(\%)$ & & & 11.934 & 0.001 \\
\hline No & $93(23.97)$ & $3(4.76)$ & & \\
\hline Yes & $295(76.03)$ & $60(95.24)$ & & \\
\hline$\geq 3$ & $182(7.22)$ & $41(65.08)$ & & \\
\hline NRDS, n (\%) & & & 55.087 & $<0.001$ \\
\hline No & $360(92.78)$ & $38(60.32)$ & & \\
\hline Yes & $28(7.22)$ & $25(39.68)$ & & \\
\hline Neonatal asphyxia, $\mathrm{n}(\%)$ & & & 30.763 & $<0.001$ \\
\hline No & $220(56.70)$ & $12(19.05)$ & & \\
\hline Yes & $168(43.30)$ & $51(80.95)$ & & \\
\hline Anemia of prematurity, $\mathrm{n}(\%)$ & & & 1.419 & 0.234 \\
\hline Clinical septicemia, n (\%) & & & 20.629 & $<0.001$ \\
\hline No & $302(77.84)$ & $32(50.79)$ & & \\
\hline Yes & $86(22.16)$ & $31(49.21)$ & & \\
\hline Pneumorrhagia, n (\%) & & & 8.961 & 0.003 \\
\hline No & $370(95.36)$ & $54(85.71)$ & & \\
\hline Yes & $18(4.64)$ & $9(14.29)$ & & \\
\hline PDA, n (\%) & & & 4.871 & 0.027 \\
\hline No & 346 (89.18) & $50(79.37)$ & & \\
\hline Yes & $42(10.82)$ & $13(20.63)$ & & \\
\hline
\end{tabular}

Table 1 (continued) 
Table 1 (continued)

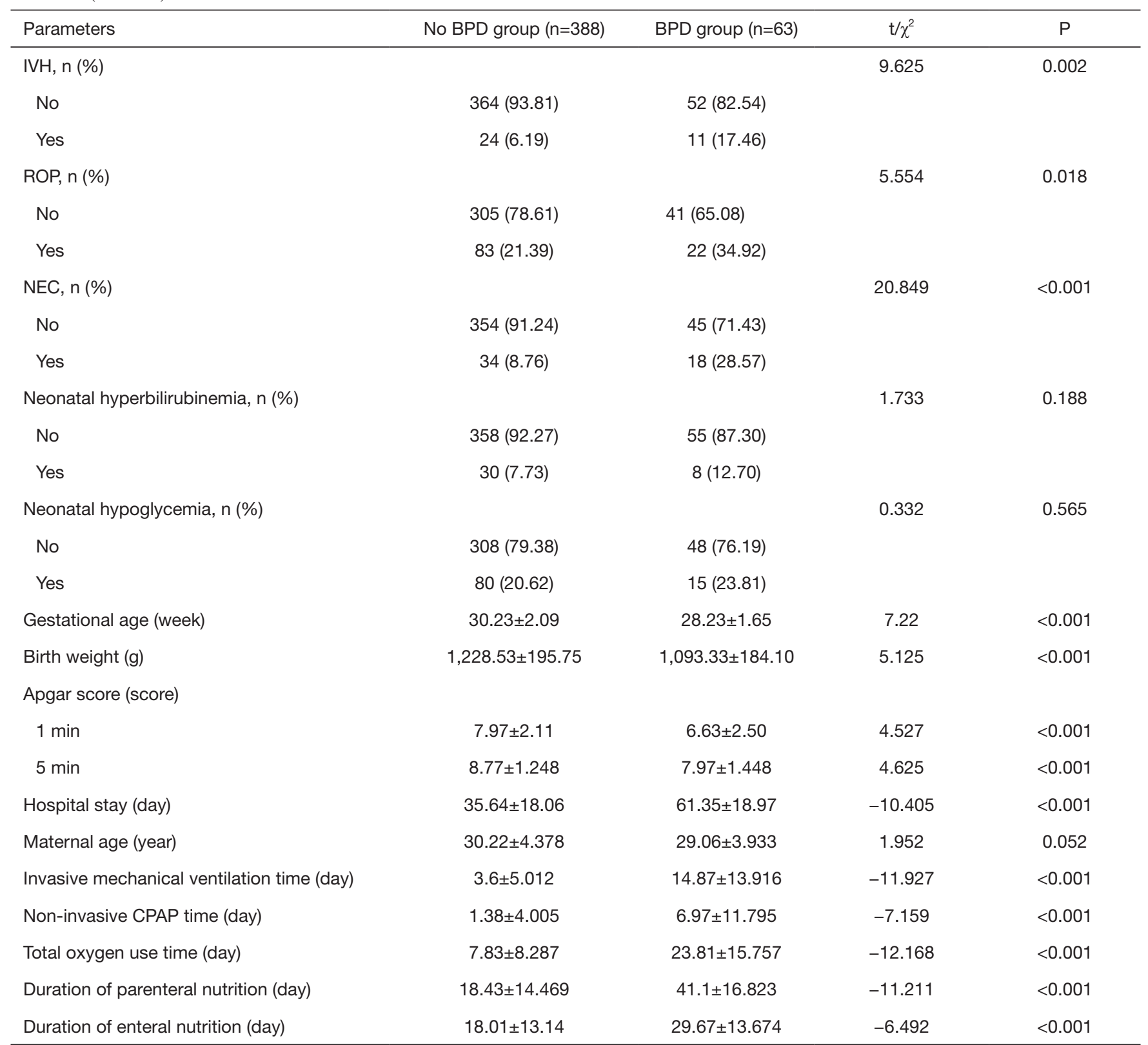

BPD, bronchopulmonary dysplasia; VLBWIs, very low birth weight infants; SGA, small for gestational age; PS, pulmonary surfactant; NRDS, neonatal respiratory distress syndrome; PDA, patent ductus arteriosus; IVH, intraventricular hemorrhage; ROP, retinopathy of prematurity; NEC, necrotizing enterocolitis; CPAP, continuous positive airway pressure.

effect of the prediction model was good.

\section{Independent data validation of the risk prediction model}

In this study, 160 infants were selected as independent data to verify the prediction effect of the model, including 86
(53.8\%) males and 74 (46.3\%) females, with gestational age at birth $29.84 \pm 2.11$ weeks and birth weight $1,211.56 \pm 179.08$ g. According to the prediction model formula in this study, the data of the 160 infants were interpolated into the cutoff value, and when the cut-off value $\geq-1.5505$, BPD was considered to occur. The ROC curve was drawn, and the 
results showed that the area under the ROC curve was 0.89 , the sensitivity was $92.9 \%$, and the specificity was $76 \%$, as shown in Figure 2.

\section{Discussion}

Significance of constructing the BPD risk prediction model for VLBWIs

$\mathrm{BPD}$ is one of the most common complications in the

Table 2 The variable assignment method of binary logistic regression analysis on the influencing factors of BPD in VLBWIs

\begin{tabular}{ll}
\hline Variable & Assignment method \\
\hline Neonatal asphyxia & No=0; yes $=1$ \\
Positive sputum culture & No=0; yes $=1$ \\
Clinical septicemia & No=0; yes $=1$ \\
NRDS & No=0; yes $=1$ \\
Number of blood transfusions & $<3=0 ; \geq 3=1$ \\
PDA & No=0; yes $=1$ \\
Duration of parenteral nutrition & Original value \\
1 min Apgar score & Original value \\
Invasive mechanical ventilation time & $<7$ day $=0 ; \geq 7$ day $=1$ \\
Oxygen time & $<14$ day $=0 ; \geq 14=1$ \\
\hline
\end{tabular}

BPD, bronchopulmonary dysplasia; VLBWIs, very low birth weight infants; NRDS, neonatal respiratory distress syndrome; PDA, patent ductus arteriosus. treatment of premature infants, and its pathogenesis is still unclear $(16,17)$. Early prevention and timely diagnosis and treatment are key to reducing the incidence and improving the prognosis $(18,19)$. In this study, the incidence of BPD was $14.9 \%$, slightly higher than the $12.3 \%$ reported by Jensen et al. (20), which may be related to the differences in research subjects, diagnostic criteria, and evaluation methods, among other factors. The length of stay in the NICU for children with BPD is significantly prolonged, and the readmission rate after discharge is twice that of preterm infants without BPD (21). In addition, the damage to the respiratory system and nervous system caused by BPD can last until adulthood (22), which, to some extent, aggravates the suffering of children and the economic burden of families. In order to better guide clinical implementation of targeted intervention measures, and to prevent and reduce the risk of BPD, previous studies have focused on the pathogenesis and clinical risk factors of BPD (23), with a lack of studies on risk assessment tools for BPD. The indicators in the risk prediction model for BPD in VLBWIs established in this study were simple and easy to obtain, and the related factors of BPD were intuitively displayed in the formula, which was convenient and easy to operate. Therefore, this model can be applied to predict the high-risk population of BPD clinically, and targeted preventive measures can be taken, such as actively preventing and controlling nosocomial infection, reducing invasive ventilation, using non-invasive ventilation modes as much as possible, and strictly controlling blood transfusion guidelines, so as to reduce the occurrence of BPD.

Table 3 Results of binary logistics regression analysis of influencing factors of BPD in VLBW

\begin{tabular}{lcccccc}
\hline Parameters & $\mathrm{B}$ & $\mathrm{SE}$ & Wald & $\mathrm{P}$ & Exp (B) & $95 \% \mathrm{Cl}$ \\
\hline Neonatal asphyxia & 1.229 & 0.535 & 5.278 & 0.022 & 3.419 & $1.198-9.757$ \\
Positive sputum culture & 1.265 & 0.554 & 5.203 & 0.023 & 3.541 & $1.195-10.497$ \\
Clinical septicemia & 1.677 & 0.489 & 11.745 & 0.001 & 5.351 & $2.05-13.963$ \\
NRDS & 1.848 & 0.605 & 9.33 & 0.002 & 6.35 & $1.939-20.792$ \\
Number of blood transfusion $(\geq 3)$ & 1.455 & 0.507 & 8.251 & 0.004 & 4.285 & $1.588-11.568$ \\
PDA & 1.835 & 0.679 & 7.308 & 0.007 & 6.266 & $1.656-23.704$ \\
1 min Apgar score & -0.25 & 0.11 & 5.156 & 0.023 & 0.778 & $0.627-0.966$ \\
Invasive mechanical ventilation time & 0.123 & 0.027 & 20.272 & 0.000 & 1.13 & $1.072-1.192$ \\
Oxygen time & 0.09 & 0.019 & 23.144 & 0.000 & 1.095 & $1.055-1.136$ \\
Duration of parenteral nutrition & 0.057 & 0.019 & 9.035 & 0.003 & 1.059 & $1.02-1.099$ \\
Constant & -8.077 & 1.408 & 32.906 & & & \\
\hline
\end{tabular}

NRDS, neonatal respiratory distress syndrome; PDA, patent ductus arteriosus. 


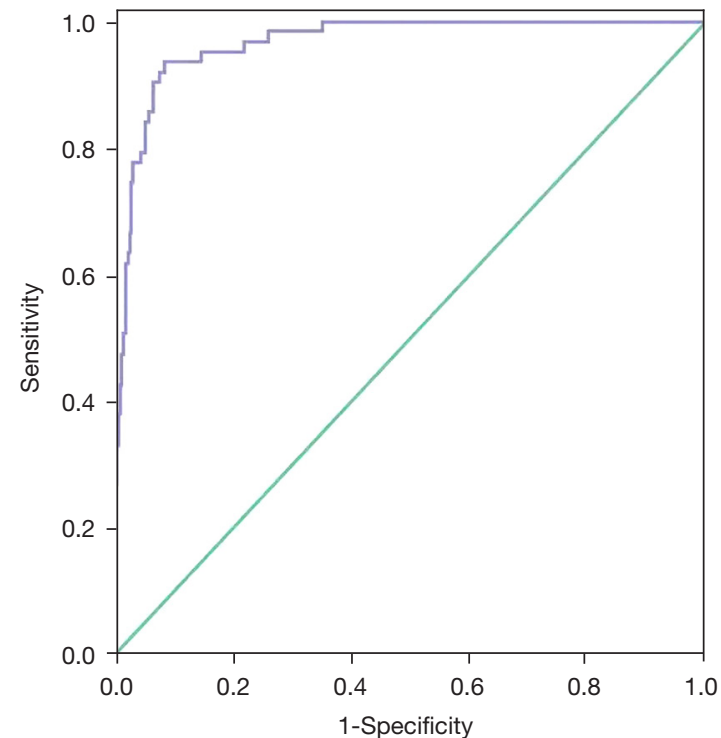

Figure 1 The ROC curve for predicting the risk of BPD in VLBWIs of the model building group. ROC, receiver operating characteristic; BPD, bronchopulmonary dysplasia; VLBWIs, very low birth weight infants.

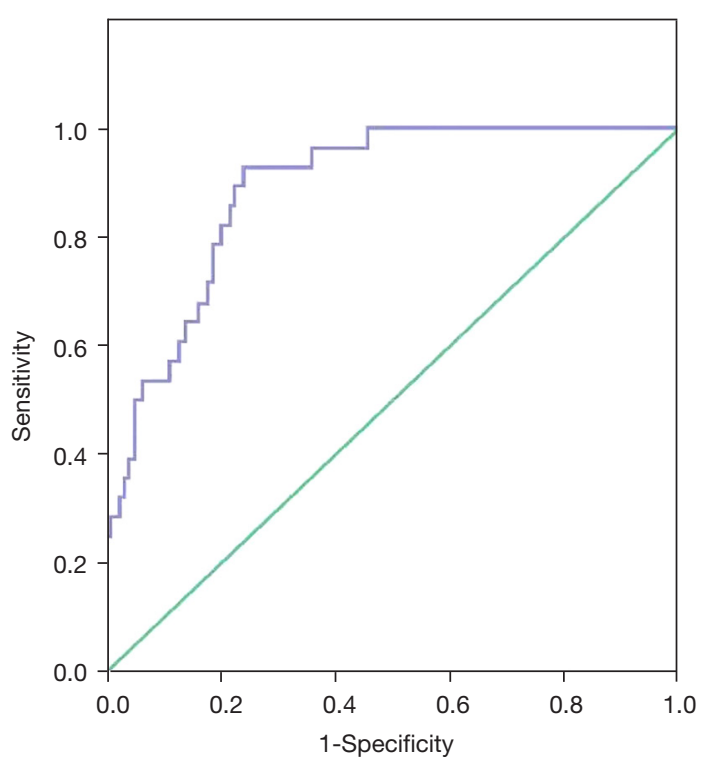

Figure 2 The ROC curve for predicting BPD risk in VLBWIs of the model validation group. ROC, receiver operating characteristic; BPD, bronchopulmonary dysplasia; VLBWIs, very low birth weight infants.

\section{Analysis of risk factors for BPD in VLBWIs}

In this study, logistic regression analysis showed that neonatal asphyxia, positive sputum culture, clinical septicemia, NRDS, blood transfusion $\geq 3$ times, PDA, invasive mechanical ventilation time, oxygen time, and parenteral nutrition time were independent risk factors. The main analysis was as follows.

Infants with immature lung development are prone to BPD (24-26). The results of this study showed that infants with a history of neonatal asphyxia and NRDS were at higher risk of developing BPD, consistent with the results of several previous studies. In terms of causes, for VLBWIs born SGA and low body weight, which results in immature lung development and difficulty in postoperative respiratory establishment, asphyxia can easily occur, and resuscitation is difficult. The occurrence of NRDS leads to an increase in PS consumption. The infant requires assisted breathing support and has difficulty stopping oxygen support for a short period of time, which to some extent aggravates lung injury and makes the infant prone to BPD. Therefore, medical personnel should improve midwifery technology to prevent neonatal asphyxia. For premature infants with immature lung development, therapeutic PS should be given as early as possible to promote lung development and maturation, prevent further deterioration of the disease, and reduce the incidence of $\mathrm{BPD}$.

The longer the time of invasive mechanical ventilation and oxygen application, the higher the susceptibility to BPD. The results of this study showed that the duration of invasive mechanical ventilation in the BPD group was longer than that in the non-BPD group, which was consistent with previous results (27). Prolonged mechanical ventilation is likely to cause mechanical and biological injuries of the lungs such as barometric injury, volume injury, oxygen toxicity injury, and atelectasis, leading to alveolar damage, pulmonary fibrosis, airway inflammation, further aggravation of hypoxia, and the formation of a vicious cycle, resulting in the occurrence of BPD. The duration of oxygen application was significantly higher in the BPD group than in the non-BPD group. Prolonged oxygen inhalation not only reduces the activity of the PS, leading to pulmonary fibrin deposition, pulmonary edema, and inflammatory reactions, among other processes, but also forms a large number of highly active oxygen free radicals in the body, which cause severe oxidative stress, inflammatory responses, alveolar wall thinning, alveolar and capillary reduction, and other lung developmental stagnation changes, which eventually lead to BPD. Premature infants are more susceptible to prolonged oxygen inhalation due to an immature antioxidant enzyme system and a limited ability to scavenge oxygen free radicals. Therefore, in 
clinical treatment, a protective lung ventilation strategy should be adopted according to the condition of the infants, in order to minimize the use of invasive ventilation, shorten the duration of ventilation, and avoid long-term oxygen therapy and repeated machine use, which is helpful to reduce the occurrence of $\mathrm{BPD}$.

Infants with postnatal infection are prone to BPD. The autoimmune function of VLBWIs is low, along with resistance to microorganisms. The condition of these infants is also critical and requires prolonged hospitalization, which increases the risk of infection. Both positive sputum culture results and clinical septicemia indicate postnatal infection, and both were independent risk factors for BPD. Many clinical observational studies have shown that postnatal infection is a risk factor for BPD (23). Infection can cause a systemic inflammatory response which affects pulmonary vascular permeability, prevents alveolar maturation of premature lungs in premature infants, further aggravates lung injury, and leads to BPD. Studies have shown that postnatal infection has a higher predictive effect for BPD than intrauterine infection (28). Cytokines, like IL-1 $\beta$, IL6 , IL-8, IL-10, IL-17, RANTES, TNF- $\alpha$ and interferon- $\gamma$, are dysregulated in the serum in VLBWIs with BPD, which indicated the transition from innate immune to adaptive immune (29). Therefore, medical staff should pay attention to postnatal infection and actively take measures to control infection, including strict aseptic operation and encouraging breastfeeding to improve immunity.

Other aspects. The results of this study showed that the risk of BPD in infants with a history of PDA was 6.266 times higher than that in infants without a history of PDA. In terms of the causes, the continuous left-right shunt after the opening of the ductus arteriosus leads to the increase of pulmonary blood flow, the decrease of pulmonary function, and the decrease of gas exchange, which leads to injuryinduced hypertrophy of the pulmonary vasculature and the aggravation of pulmonary inflammation. Meanwhile, it also leads to an increase in the demand for mechanical ventilation, difficulty in ventilator withdrawal, and an increased risk of BPD. Therefore, patients with BPD combined with PDA should receive surgical treatment as soon as possible to close the ductus arteriae. Studies have shown that the greater the amount of blood transfusion during hospitalization, the higher the risk of $\mathrm{BPD}(30)$. The results of this study showed that the risk of BPD was 4.285 times higher in infants with $\geq 3$ transfusions than in infants with $<3$ transfusions. This may be related to permanent changes in lung tissue due to increased oxidative stress damage caused by blood transfusion, which increases the risk of BPD. Therefore, iatrogenic anemia should be avoided as much as possible, umbilical cord ligation should be delayed, and blood transfusion indications should be strictly mastered. In addition, the results of this study also showed that the number of days of parenteral nutrition was an independent risk factor for BPD. Intravenous nutrient solution contains a variety of hepatotoxic amino acids, and fat emulsions will produce excessive lipid peroxides during metabolism, which will not only cause liver damage, but also induce or aggravate BPD $(31,32)$. Enteral nutrition should be established as soon as possible to ensure the normal development of lung tissue.

\section{The predictive effect of the $V L B W I B P D$ risk prediction model is good}

This study finally constructed a prediction model with 10 indicators that are easy to evaluate as variables. Clinical staff can assess the risk of BPD in VLBWIs with relative ease. An area under the ROC curve value between 0.5 and 0.7 indicates that the discriminant efficiency of the model is low, 0.7-0.9 indicates medium discriminant efficiency, while $0.9-1.0$ indicates high discriminant efficiency (33). The area under the ROC curve of the model in this study is 0.965 , indicating that the model has good prediction efficiency for the occurrence of BPD in VLBWIs. Applying the model to clinical practice, the results showed that the area under the ROC curve was 0.89 , the sensitivity was $92.9 \%$, and the specificity was $76 \%$, indicating that the model had good predictive performance and was beneficial to the screening of VLBWIs with BPD risk.

\section{Innovation and limitation of this research}

This study attempted to build a risk prediction model to determine whether BPD occurs in VLBWIs. No relevant research has yet been reported. In addition, the prediction model is simple and it is convenient to obtain data, which can provide a reference for the clinical prevention and treatment of BPD. The limitation of this study lies in the small number of research samples, and the study was conducted in only one tertiary grade A hospital. In the future, multi-center and large-sample studies can be conducted to further revise and improve the applicability of the model, so as to facilitate its later application in clinical practice. 


\section{Conclusions}

In this study, 10 independent risk factors for BPD in VLBWIs were screened out by univariate and multivariate logistic regression analyses, and the risk prediction model was established by data analysis. The model has good predictive ability and can predict the occurrence of BPD in VLBWIs robustly, which provides a basis for clinical work and has good clinical application value.

\section{Acknowledgments}

Funding: Maternal and Child Health Research Project of Jiangsu Province (F201936); Basic Research on the People's Livelihood Science and Technology-Medical Health in Suzhou Science and Technology Bureau (SYS2020039).

\section{Footnote}

Reporting Checklist: The authors have completed the STARD reporting checklist. Available at https://dx.doi. org/10.21037/tp-21-445

Data Sharing Statement: Available at https://dx.doi. org/10.21037/tp-21-445

Conflicts of Interest: All authors have completed the ICMJE uniform disclosure form (available at https://dx.doi. org/10.21037/tp-21-445). The authors have no conflicts of interest to declare.

Ethical Statement: The authors are accountable for all aspects of the work in ensuring that questions related to the accuracy or integrity of any part of the work are appropriately investigated and resolved. All procedures performed in this study involving human participants were in accordance with the Declaration of Helsinki (as revised in 2013). The study was approved by ethics committee of Suzhou Municipal Hospital (No.: K-2020-053-K01) and informed consent was taken from the patients' parents or legal guardians.

Open Access Statement: This is an Open Access article distributed in accordance with the Creative Commons Attribution-NonCommercial-NoDerivs 4.0 International License (CC BY-NC-ND 4.0), which permits the noncommercial replication and distribution of the article with the strict proviso that no changes or edits are made and the original work is properly cited (including links to both the formal publication through the relevant DOI and the license). See: https://creativecommons.org/licenses/by-nc-nd/4.0/.

\section{References}

1. Principi N, Di Pietro GM, Esposito S. Bronchopulmonary dysplasia: clinical aspects and preventive and therapeutic strategies. J Transl Med 2018;16:36.

2. Ballard AR, Mallett LH, Pruszynski JE, et al. Chorioamnionitis and subsequent bronchopulmonary dysplasia in very-low-birth weight infants: a 25 -year cohort. J Perinatol 2016;36:1045-8.

3. Kandasamy J, Roane C, Szalai A, et al. Serum eotaxin-1 is increased in extremely-low-birth-weight infants with bronchopulmonary dysplasia or death. Pediatr Res 2015;78:498-504.

4. Sharma A, Xin Y, Chen X, et al. Early prediction of moderate to severe bronchopulmonary dysplasia in extremely premature infants. Pediatr Neonatol 2020;61:290-9.

5. Valenzuela-Stutman D, Marshall G, Tapia JL, et al. Bronchopulmonary dysplasia: risk prediction models for very-low- birth-weight infants. J Perinatol 2019;39:1275-81.

6. Klinger G, Sokolover N, Boyko V, et al. Perinatal risk factors for bronchopulmonary dysplasia in a national cohort of very-low-birthweight infants. Am J Obstet Gynecol 2013;208:115.e1-9.

7. Foglia EE, Jensen EA, Kirpalani H. Delivery room interventions to prevent bronchopulmonary dysplasia in extremely preterm infants. J Perinatol 2017;37:1171-9.

8. Li WL, Xu FL, Niu M, et al. Clinical features and prognosis of preterm infants with varying degrees of bronchopulmonary dysplasia. Zhongguo Dang Dai Er Ke Za Zhi 2018;20:261-6.

9. Laughon MM, Langer JC, Bose CL, et al. Prediction of bronchopulmonary dysplasia by postnatal age in extremely premature infants. Am J Respir Crit Care Med 2011;183:1715-22.

10. Jain D, Bancalari E. Bronchopulmonary dysplasia: clinical perspective. Birth Defects Res A Clin Mol Teratol 2014;100:134-44.

11. Bhandari A, Bhandari V. Pitfalls, problems, and progress in bronchopulmonary dysplasia. Pediatrics 2009;123:1562-73.

12. Check J, Gotteiner N, Liu X, et al. Fetal growth restriction and pulmonary hypertension in premature infants with bronchopulmonary dysplasia. J Perinatol 2013;33:553-7. 
13. Lal CV, Olave N, Travers C, et al. Exosomal microRNA predicts and protects against severe bronchopulmonary dysplasia in extremely premature infants. JCI Insight 2018;3:e93994.

14. Janér J, Andersson S, Kajantie E, et al. Endostatin concentration in cord plasma predicts the development of bronchopulmonary dysplasia in very low birth weight infants. Pediatrics 2009;123:1142-6.

15. Peduzzi P, Concato J, Feinstein AR, et al. Importance of events per independent variable in proportional hazards regression analysis. II. Accuracy and precision of regression estimates. J Clin Epidemiol 1995;48:1503-10.

16. Hwang JS, Rehan VK. Recent Advances in Bronchopulmonary Dysplasia: Pathophysiology, Prevention, and Treatment. Lung 2018;196:129-38.

17. Piersigilli F, Bhandari V. Metabolomics of bronchopulmonary dysplasia. Clin Chim Acta 2020;500:109-14.

18. Gursoy T, Hayran M, Derin H, et al. A clinical scoring system to predict the development of bronchopulmonary dysplasia. Am J Perinatol 2015;32:659-66.

19. Philpot PA, Bhandari V. Predicting the likelihood of bronchopulmonary dysplasia in premature neonates. Expert Rev Respir Med 2019;13:871-84.

20. Jensen EA, Schmidt B. Epidemiology of bronchopulmonary dysplasia. Birth Defects Res A Clin Mol Teratol 2014;100:145-57.

21. Abman SH, Collaco JM, Shepherd EG, et al. Interdisciplinary Care of Children with Severe Bronchopulmonary Dysplasia. J Pediatr 2017;181:12-28.e1.

22. Kotecha S, Clemm H, Halvorsen T, et al. Bronchial hyperresponsiveness in preterm-born subjects: A systematic review and meta-analysis. Pediatr Allergy Immunol 2018;29:715-25.

23. Clinical characteristics and risk factors of very low birth weight and extremely low birth weight infants with bronchopulmonary dysplasia: multicenter retrospective analysis. Zhonghua Er Ke Za Zhi 2019;57:33-9.

24. Cokyaman T, Kavuncuoglu S. Bronchopulmonary dysplasia frequency and risk factors in very low birth

Cite this article as: Cai H, Jiang L, Liu Y, Shen T, Yang Z, Wang S, Ma Y. Development and verification of a risk prediction model for bronchopulmonary dysplasia in very low birth weight infants. Transl Pediatr 2021;10(10):2533-2543. doi: 10.21037/ tp-21-445 weight infants: A 3-year retrospective study. North Clin Istanb 2020;7:124-30.

25. Short EJ, Klein NK, Lewis BA, et al. Cognitive and academic consequences of bronchopulmonary dysplasia and very low birth weight: 8 -year-old outcomes. Pediatrics 2003;112:e359.

26. Isayama T, Lee SK, Yang J, et al. Revisiting the Definition of Bronchopulmonary Dysplasia: Effect of Changing Panoply of Respiratory Support for Preterm Neonates. JAMA Pediatr 2017;171:271-9.

27. Jensen EA, Dysart K, Gantz MG, et al. The Diagnosis of Bronchopulmonary Dysplasia in Very Preterm Infants. An Evidence-based Approach. Am J Respir Crit Care Med 2019;200:751-9.

28. Lahra MM, Beeby PJ, Jeffery HE. Intrauterine inflammation, neonatal sepsis, and chronic lung disease: a 13-year hospital cohort study. Pediatrics 2009;123:1314-9.

29. Ambalavanan N, Carlo WA, D'Angio CT, et al. Cytokines associated with bronchopulmonary dysplasia or death in extremely low birth weight infants. Pediatrics 2009;123:1132-41.

30. Ghirardello S, Dusi E, Cortinovis I, et al. Effects of Red Blood Cell Transfusions on the Risk of Developing Complications or Death: An Observational Study of a Cohort of Very Low Birth Weight Infants. Am J Perinatol 2017;34:88-95.

31. Shen K, Feng X, Pan H, et al. Baicalin Ameliorates Experimental Liver Cholestasis in Mice by Modulation of Oxidative Stress, Inflammation, and NRF2 Transcription Factor. Oxid Med Cell Longev 2017;2017:6169128.

32. Xu Z, Sun Y. The Role of Parenteral Lipids in the Development of Hepatic Dysfunction and Hepatic Steatosis in a Mouse Model of Total Parenteral Nutrition. J Nutr Sci Vitaminol (Tokyo) 2019;65:24-30.

33. Chen Y, Du H, Wei BH, et al. Development and validation of risk-stratification delirium prediction model for critically ill patients: A prospective, observational, singlecenter study. Medicine (Baltimore) 2017;96:e7543.

(English Language Editor: C. Betlzar) 